\title{
Exploiting ternary blends for improved photostability in high efficiency organic solar cells
}

Nicola Gasparini*, Sri Harish Kumar Paleti, Jules Bertrandie, Guilong Cai, Guichuan Zhang, Andrew Wadsworth, Xinhui Lu, Hin-Lap Yip, Iain McCulloch, Derya Baran*

Dr. N. Gasparini, S.H.K. Paleti, J. Bertrandie, Prof. D. Baran

King Abdullah University of Science and Technology (KAUST), Division of Physical Sciences and Engineering (PSE), KAUST Solar Center (KSC), Thuwal, 23955, Saudi Arabia

Dr. Guilong Cai, Prof. Xinhui Lu

Department of Physics, The Chinese University of Hong Kong, New Territories, Hong Kong

Dr. Guichuan Zhang, Prof. Hin-Lap Yip

Institute of Polymer Optoelectronic Materials and Devices, State Key Laboratory of Luminescent Materials and Devices, School of Materials Science and Engineering, South China University of Technology, Guangzhou, 510640, China

Dr. A. Wadsworth, Prof. I. McCulloch

Imperial College London, Department of Chemistry, 80 Wood Lane, London, W12 0BZ, UK

Corresponding author email: n.gasparini@imperial.ac.uk, derya.baran@kaust.edu.sa 


\begin{abstract}
Ternary organic solar cells based on polymer donor and nonfullerene acceptors (NFAs) are delivering high power conversion efficiencies (PCE). Now, further improvement needs to be directed to enhance the operational lifetime of organic photovoltaics. Here, we selected three NFAs with different electron affinities and structural properties and found that the most crystalline third component, O-IDTBR, is selectively miscible within the acceptor phase. This reduced trap-assisted recombination and delivered a PCE of $16.6 \%$ and a fill factor of 0.76 , compared to PM6:Y6 binary devices $(15.2 \%$ PCE). Charge transport and recombination analyses revealed that O-IDTBR acts as a charge relay for improved charge transfer of both donor and acceptor materials leading to a more ordered transport. We find that minimizing traps formation in ternary devices deactivates light-induced traps upon full sun illumination (AM1.5G). As a result, ternary devices do not show any PCE drop in $225 \mathrm{~h}$, in comparison to binary cells which lose more than $60 \%$ of their initial performances.
\end{abstract}


Organic photovoltaics (OPV), in which the photoactive bulk-heterojunction (BHJ) layer consists of donor polymers and nonfullerene acceptors (NFAs), are reaching power conversion efficiencies (PCE) in the vicinity of their inorganic counterparts. ${ }^{1-8}$ Among the pool of strategies for improving the PCE in OPVs, the ternary concept consisting of blending multiple donors and/or acceptors, while maintaining the single-junction configuration, is the main driving force towards this record performances. ${ }^{9-14}$ Currently, PCE over $16.5 \%$ are reported for ternary blends, ${ }^{15}$ which challenges the more complex multi-junction configurations. ${ }^{16-18}$ The field has been driven by the development of ultra-low narrow bandgap NFAs (such as IEICO-4F and Y6) in combination with middle/wide band gap polymer donors (such as PTB7-Th and PBDB-T-2F) with high photocurrent and photovoltage..$^{719-21}$ These highly efficient blends are potential candidates to further push the efficiency threshold of OPV, where non-radiative voltage losses as well as FF limitations are still a challenge. ${ }^{10,22,23}$ So far, highly performing OPV devices are usually not combined with long-term photostability. ${ }^{24,25}$ With the development of the PM6:Y6 blend, it has been possible to reach record efficiencies of over $16 \% .{ }^{19}$ At present, however, there has been significantly less focus on the stability of Y6-based devices.

Light-induced traps formation has been the cause of open-circuit voltage $\left(V_{\text {oc }}\right)$ losses in several fullerene and NFA-based devices. ${ }^{26,27}$ Severe burn-in in PCE has been observed for hypo-miscible donor-acceptor blends, which leads to a spontaneous demixing, even at room temperature. $^{28}$ Moreover, the crystallization of NFA in the blend film can render morphological instabilities. ${ }^{29,30}$ The ternary strategy has shown to control and reduce one or all of the limitations above compared to binary devices, yet a meticulous design and choice of the third component is crucial. ${ }^{14}$

In this work, we highlight several novel ternary solar cell systems using nonfullerene acceptors (O-IDTBR, EH-IDTBR and O-IDFBR, see Figure S1 in the Supporting Information (SI)) resulting in a maximum PCE of $16.63 \%$ where light induced traps were mitigated 
compared to binary devices for long-term photostability. Among them, the addition of a midband gap indacenodithiophene derivative NFA, O-IDTBR $(15 \% \mathrm{w}: \mathrm{w})$ as third component improves all the photovoltaic parameters in the reference binary device PM6:Y6 (15.21\% PCE) as well as the photostability. We found that O-IDTBR tends to preferentially mix only within the acceptor matrix and, at the same time operates as a charge relay for both hole and electron transfer between the binary components. The better charge transfer in ternary devices together with reduced trap-assisted recombination leads to a FF of as high as 0.76 . More importantly, upon light degradation at one Sun condition, ternary solar cells show a negligible PCE drop, whereas the binary cells lose more than $60 \%$ of their initial performances in the first $225 \mathrm{~h}$ of device operation. Finally, we revealed that the high photostability of ternary devices arises from the more tolerance towards light-induced traps, which in contrast deteriorate the binary cells longevity.

In this study, we selected three different NFAs, O-IDTBR, EH-IDTBR and O-IDFBR as potential third components owing to their different electron affinity (EA) values that can potentially contribute to open-circuit voltage $\left(V_{\text {oc }}\right)$, as well as their different molecular ordering in thin film that would potentially impact charge transport in ternary devices. The chemical structures of the donor and acceptor materials used in this study are represented in Figure 1a and Figure S1a. The energy levels calculated from photoelectron spectroscopy in air (PESA) of the materials used are presented in Figure 1b and Figure S1b-e, where the addition of O-IDTBR and EH-IDTBR represents an energy-cascade model and the corresponding thin film absorbance profiles ranging from visible to near-IR is shown in

\section{Figure 1c and Figure S1f.}

First, to elucidate the role of the third component on the thermal behavior and miscibility we performed differential scanning calorimetry (DSC) measurements for neat EH-IDTBR, OIDFBR and O-IDTBR as well as their binary acceptor and ternary blends (Figure 1d, Figure S2 and Figure S3). Figure 1d depicts the heat flow DSC profiles of neat PM6, Y6, O-IDTBR, 
binary and ternary blends. O-IDTBR has an exothermic crystallization transition with $\mathrm{T}_{\mathrm{c}}$ of $115^{\circ} \mathrm{C}$ and a melting peak around $225^{\circ} \mathrm{C}$, which is similar to previously reported values. ${ }^{31} \mathrm{~A}$ weak and broad endotherm was observed for Y6 around $200{ }^{\circ} \mathrm{C}$ and no evidence for any thermal transitions for PM6 was present (Figure 1d). In the binary Y6:O-IDTBR blend, the O-IDTBR melting endotherm is still prominent, but the peak is broadened and shows a melting point depression and lower enthalpy, additionally the broad endotherm from Y6 around $200{ }^{\circ} \mathrm{C}$ is depressed slightly. The new melting and crystallization peaks of Y6:OIDTBR binary blend were unaffected upon blending with PM6, indicating that newly formed binary O-IDTBR:Y6 phases were preserved in the ternary blend. O-IDFBR and EH-IDTBR have endotherm transitions with peaks around $185^{\circ} \mathrm{C}$ and $220^{\circ} \mathrm{C}$, respectively, similar to previously reported values. ${ }^{31}$ In the binary Y6:EH-IDTBR blend, we did not observe a peak shift, but rather a reduced melting enthalpy of EH-IDTBR (Figure S2) O-IDFBR is an amorphous NFA in thin film and can only show a strong melting peak from a concentrated solution measured by DSC measurements. ${ }^{32}$ The crystallite melt is significantly reduced and broadened upon mixing with Y6, however no melting point depression is observed (Figure S3). This picture suggests the formation of a well-mixed amorphous solid phase as well as the lack of ordered microstructure.

To reveal more about the nature of molecular ordering and orientation in the binary and ternary blends, we performed grazing incidence wide angle X-ray scattering (GIWAXS). In line with previously reported GIWAXS of neat O-IDTBR, the annular diffraction feature suggests a polycrystalline nature without preferred orientation (Figure 2). This feature is preserved in PM6:O-IDTBR binary film, especially the lamellar peak around $0.44 \AA-1$, indicating O-IDTBR prefers to self-crystallized rather than mixing with PM6. However, the distinctive scattering features of O-IDTBR are missing in the ternary, which suggests that OIDTBR molecules prefers to interact with Y6 rather than self-crystallization, upon addition of Y6. Furthermore, the addition of O-IDTBR does not significantly affect the crystallization of 
Y6, as the lamellar peak at $\sim 0.45 \AA^{-1}$ in the in-plane direction and the $(010)$ peak at $\sim 1.75 \AA^{-}$

${ }^{1}$ in the out-of-plane direction for Y6 are preserved in both binary and ternary films. The addition of $15 \mathrm{wt} \%$ O-IDTBR into PM6:Y6 binary system slightly enhances the $(010) \pi-\pi$ interaction at $\sim 1.75 \AA^{-1}$ in the out-of-plane direction, in line with the superior transport properties of the ternary blend. ${ }^{33-35}$ The intensity of the $(010)$ peak slightly decreases when the content of O-IDTBR increases to $25 \mathrm{wt} \%$, suggesting that an excess O-IDTBR will also affect the molecular $\pi-\pi$ interactions of Y6. We have previously reported that the formation of new mixed phases was beneficial for charge transport and reduced recombination in ternary NFA solar cells. ${ }^{32}$ With this in mind the O-IDTBR based ternary system was considered the most promising for further investigation.

We have calculated the Flory-Huggins interaction parameter, $\chi$ of the O-IDTBR binary combinations from contact angle measurements using the relation $\chi_{1,2} \propto\left(\sqrt{\gamma_{1}}-\sqrt{\gamma_{2}}\right)^{2}$, where 1 and 2 are the individual components in binary blends (See Figure S4 and Table S1 for further details). ${ }^{36-38}$ The $\chi$ values for PM6:Y6 and Y6:O-IDTBR binary blends were calculated as 0.24 and 0.46 , respectively, suggesting well-mixed phases between two components. However, PM6:O-IDTBR has a $\chi$ value of 1.35 which is a direct indication that PM6 and O-IDTBR have a much lower preference to mix than either Y6 with O-IDTBR or PM6 with Y6 (where a higher $\chi$ means less interaction between the two components). All of these observations support the DSC results. A ternary blend with a highly crystalline third component, selectively miscible with one of the components in the blend, would be a promising strategy to improve the charge transport and/or reducing charge recombination as previously discussed.

To test the photo-physics of the addition of O-IDTBR in PM6:Y6 binary devices, we fabricated OPV devices in a conventional device architecture (ITO/PEDOT/active layer/DPO/Ag), keeping the donor:acceptor ratio (D:A) constant. We measured the devices under 1 sun (AM1.5G) equivalent illumination and the corresponding current density-voltage 
$(J-V)$ curves are summarized in Figure 3a and Figure S5. PM6:Y6 reference binary devices delivered a PCE of $15.21 \%$ together with an open-circuit voltage $\left(V_{\text {oc }}\right)$ of $0.83 \mathrm{~V}$, a short circuit current density $\left(J_{\mathrm{sc}}\right)$ of $25.10 \mathrm{~mA} \mathrm{~cm} \mathrm{~cm}^{-2}$ and a fill factor $(\mathrm{FF})$ of 0.73 , which is comparable to reported literature. ${ }^{19}$ Upon addition of $15 \%$ by weight of O-IDTBR into PM6:Y6 blend, the ternary device displayed an enhancement of all the photovoltaic parameters, with a $V_{\mathrm{oc}}$ of $0.85 \mathrm{~V}, J_{\mathrm{sc}}$ of $25.75 \mathrm{~mA} \mathrm{~cm} \mathrm{~cm}^{-2}$ and a FF of 0.76 , resulting in a PCE of $16.63 \%$ for the best devices. Figure S6 shows the PCE distribution of binary and ternary devices. Higher loading of O-IDTBR ( $>25 \%$ by weight) leads to reduced performances (PCE of $15.11 \%)$, mainly due to deteriorated FF (0.69) compared to the PM6:Y6 1:1 and PM6:OIDTBR:Y6 1:0.15:0.85 devices. The key photovoltaic parameters for binary and ternary devices are summarized in Table 1. Based on the energy level measurements, one of the advantages of O-IDTBR is its decreased EA value compared to Y6 (creating an energy cascade) which could contribute to $V_{o c}$ in ternary devices compared to binary ones. Although the EA values between the two acceptors have about $160 \mathrm{mV}$ difference, there is only an incremental increase in $V_{o c}$ values in ternary devices upon addition of $15 \%$ O-IDTBR $(\sim 20$ $\mathrm{mV}$ ), which is not common for energy cascade model ternary systems. Until $30 \%$ addition of O-IDTBR, the $V_{o c}$ the ternary devices showed similar values to the binary devices (Figure S7). These observations suggest that the energy cascade model in ternary devices is much more complex than just comparing the energetic levels of the donors or acceptors. In this ternary system, it is clear from DSC results that O-IDTBR preferably mixes with Y6 forming new mixed phases. As such, the improvement in $V_{o c}$ is less significant than would be expected based on the EA values of the NFAs. Thus, we explain this abnormal phenomenon using surface energy measurements where interaction parameters are calculated (Figure S4), suggesting that O-IDTBR is well blended with Y6 to form new phases and residing mostly in the acceptor phase and at the Y6/O-IDTBR interface and not predominantly mixing with the donor phase. In comparison, ternary blends based on PM6:EH-IDTBR:Y6 (1:0.1:0.9) (Figure 
S8) delivered a PCE of $15.5 \%$ with a slightly higher $J_{\mathrm{sc}}$ of $25.9 \mathrm{~mA} \mathrm{~cm} \mathrm{~cm}^{-2}$ compared to PM6:Y6 binary devices $\left(25.1 \mathrm{~mA} \mathrm{~cm}^{-2}\right)$ and a FF of 0.71 , which is similar to binary devices. The lack of formation of new mixed phases observed in DSC profiles may be the reason for no significant improvement in FF. PM6:O-IDFBR:Y6 (1:0.1:0.9) ternary devices benefited from the shallower EA of O-IDFBR (3.7 eV) (Figure S1b) compared to O-IDTBR. This delivered a PCE of $16.2 \%$ along with a Voc of $0.89 \mathrm{~V}$ (Figure S9). However, the nature of disordered microstructure in thin film form translates into a retained FF (0.72) in PM6:OIDFBR:Y6 (1:0.1:0.9) ternary solar cells (Table 1).

To further gain insight on the contribution of the O-IDTBR into the light-to-current conversion, we first carried out external quantum efficiency (EQE) measurements (Figure 3b). The integrated current density matches the measured $J_{\mathrm{sc}}$ with a margin of less than $5 \%$ (Figure S8b, S9b and S10). The addition of O-IDTBR - for both $15 \%$ and $25 \%$ compositions - increased the $E Q E$ for all wavelength range from visible to near-IR, compared to the binary devices. This can result from a perfect ladder-type energy level between the acceptors, in which O-IDTBR acts a charge relay for both hole and electron transfer. ${ }^{39}$

We recently demonstrated that charge transfer mechanisms can be revealed by fabricating hole and electron only bilayer devices. ${ }^{35,40}$ In this study, the bilayer diodes for hole only and electron only devices are constructed based on ITO/PEDOT/Y6/O-IDTBR/PEDOT/Ag and ITO/ZnO/Y6/O-IDTBR/ZnO/Ag, respectively. Figure 3c shows the asymmetric behavior of $J-V$ curves for both bilayer configurations, confirming the hole transfer from Y6 to O-IDTBR and vice versa. These results are in line with the energy levels depicted in Figure $\mathbf{1 b}$ and the reason of the enhanced $E Q E$ in the all spectral range in ternary devices. We subsequently focused on the impact of O-IDTBR on the charge recombination dynamics in the ternary blends. The facilitated charge transfer upon addition of the third component can extract charges trapped in both donor and/or acceptor moieties. ${ }^{35}$ As a first and facile indication, we measured the $J-V$ characteristics at different light intensities for binary and ternary devices 
(Figure S11). The plot of $V_{\text {oc }}$ vs. the natural logarithm of the light intensity provides information about trap-assisted recombination. A slope of $2 k \mathrm{~T} / q$ is often associated to traps, whereas a slope of $k \mathrm{~T} / q$ is observed for bimolecular recombination. ${ }^{41}$ The calculated slopes for PM6:Y6, PM6:O-IDTBR:Y6 1:0.15:0.85 and PM6:O-IDTBR:Y6 1:0.25:0.75 are 1.12 $k \mathrm{~T} / q, 1.03 \mathrm{kT} / q$ and $1.11 \mathrm{kT} / q$, respectively, confirming the reduced trap assisted recombination in the best ternary devices (Figure 3d). Then, we studied the capability of binary and ternary devices for charge generation and extraction in both steady-state and transient measurements starting with photocurrent density $\left(J_{\mathrm{ph}}, J_{\mathrm{ph}}=J_{\mathrm{L}}-\mathrm{J}_{\mathrm{D}}\right.$, where $J_{\mathrm{L}}$ and $J_{\mathrm{D}}$ are the current density in the light and in the dark, respectively) as a function of the effective voltage ( $V_{\text {eff }}, V_{\text {eff }}=V_{0^{-}} V$, where $V_{0}$ is the displacement voltage). ${ }^{42,43}$ In all devices $J_{\text {ph }}$ quickly saturates at $V_{\text {eff }}<1 \mathrm{~V}$, suggesting that all photogenerated charges are likely to dissociate and be collected at the electrodes (Figure 4a). In the saturated regime we are able to calculate the maximum generation rate of free charges $\left(\mathrm{G}_{\max }\right)$ according to $J_{\text {sat }}=q \mathrm{G}_{\max } \mathrm{d}$, where $\mathrm{d}$ is the active layer thickness. The $\mathrm{G}_{\max }$ calculated for PM6:Y6, PM6:O-IDTBR:Y6 1:0.15:0.85 and PM6:O-IDTBR:Y6 1:0.25:0.75 devices are $1.72 \times 10^{-22} \mathrm{~cm}^{-3} \mathrm{~s}^{-1}, 1.87 \times 10^{-22} \mathrm{~cm}^{-3} \mathrm{~s}^{-1}$ and $1.81 \times 10^{-22} \mathrm{~cm}^{-3} \mathrm{~s}^{-1}$ respectively, in line with the $J_{\mathrm{sc}}$ values calculated under 1 sun illumination. We observe a similar trend at the maximum power point $\left(\mathrm{G}_{\mathrm{MPP}}\right)$ in which the charge generation rate achieved $72 \%, 80 \%$ and $67 \%$ of $\mathrm{G}_{\max }$ for PM6:Y6, PM6:O-IDTBR:Y6 1:0.15:0.85 and PM6:O-IDTBR:Y6 1:0.25:0.75 devices. In line with the higher $J_{\text {sc }}$ values obtained for ternary blend, we observe improved charge extraction behavior upon addition of 15\% O-IDTBR (Figure 4b) using transient photocurrent (TPC) measurements. Interestingly, the reduced trap-assisted recombination in PM6:O-IDTBR:Y6 1:0.15:0.85 ternary blends is reflected mainly in charge extraction, whereas the charge generation in the early time scale $\left(200 \mu\right.$ s) remains similar between binary and ternary devices (Figure S12) ${ }^{44}$

A widely used technique which allows the concomitant calculation of charge carrier mobility $(\mu)$ and bimolecular recombination coefficient $(\beta)$ is photoinduced charge carrier extraction 
by linearly increasing voltage (photo-CELIV). ${ }^{45}$ The photo-CELIV traces for binary and ternary devices at fixed delay time $\left(t_{d}\right)$ allow us to calculate the $\mu$ values as $8.5 \times 10^{-4} \mathrm{~cm}^{2} \mathrm{~V}^{-1} \mathrm{~s}^{-}$ ${ }^{1}, 1.2 \times 10^{-3} \mathrm{~cm}^{2} \mathrm{~V}^{-1} \mathrm{~s}^{-1}$ and $5.2 \times 10^{-4} \mathrm{~cm}^{2} \mathrm{~V}^{-1} \mathrm{~s}^{-1}$ (Table 2) for PM6:Y6, PM6:O-IDTBR:Y6 1:0.15:0.85 and PM6:O-IDTBR:Y6 1:0.25:0.75 solar cells, respectively (Figure S13). The high $\mu$ obtained upon addition of $15 \%$ O-IDTBR is in well agreement with the high FF (0.76). Moreover, this exceptionally high mobility for the ternary blend should resemble an ordered charge transport. Figure $\mathbf{4 c}$ depicts the electric-field-dependent charge mobility obtained by varying the highest extraction voltage from photo-CELIV measurements. PM6:O-IDTBR:Y6 1:0.15:0.85 exhibits $\mu$ values independent from the applied electric field, whereas the binary and PM6:O-IDTBR:Y6 1:0.25:0.75 solar cells show a field-dependent mobility, which are finger prints of dispersive transport and therefore reduced FF. To underpin the recombination dynamics, and in particular to calculate $\beta$, we measured photo-CELIV at different time delays $\left(t_{\mathrm{d}}\right)$ (Figure S14) ${ }^{46,47}$ In solar cells characterized by pure bimolecular recombination the charge density $(n)$ as a function of $t_{\mathrm{d}}$ should follow the equation $\mathbf{1}$ :

$$
n(t)=\frac{n_{0}}{1+n_{0} \beta t}
$$

where $n(\mathrm{t})$ is the charge density at time $t$ and $\mathbf{n}_{0}$ is the initial charge density. Figure $4 \mathbf{d}$ shows $\mathrm{n}$ vs $t_{\mathrm{d}}$ for binary and ternary devices which fits well equation 1 . The calculated $\beta$ values for

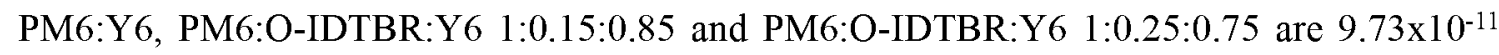
$\mathrm{cm}^{3} \mathrm{~s}^{-1}, 4.75 \times 10^{-11} \mathrm{~cm}^{3} \mathrm{~s}^{-1}$ and $1.38 \times 10^{-10} \mathrm{~cm}^{3} \mathrm{~s}^{-1}$, respectively. According to the Langevin model, the recombination rate of hole and electrons can be described by the Langevin recombination coefficient $\left(\beta_{\mathrm{L}}\right)$, defined as $\beta_{L}=\frac{q *\left(\mu_{h}+\mu_{g}\right)}{s_{0} * \varepsilon}$, where $\mu_{\mathrm{h}}$ and $\mu_{\mathrm{e}}$ are the mobility of holes and electrons, respectively, $\varepsilon_{0}$ is the vacuum and $\varepsilon$ the relative permittivity. ${ }^{48} \mathrm{We}$ calculate $\beta_{\mathrm{L}}$ of $5.13 \times 10^{-10} \mathrm{~cm}^{3} \mathrm{~s}^{-1}, 7.24 \times 10^{-10} \mathrm{~cm}^{3} \mathrm{~s}^{-1}$ and $3.14 \times 10^{-10} \mathrm{~cm}^{3} \mathrm{~s}^{-1}$ for PM6:Y6, PM6:O-IDTBR:Y6 1:0.15:0.85 and PM6:O-IDTBR:Y6 1:0.25:0.75 devices, respectively. Due to the low mobility and dispersive transport, organic solar cells are often described by 
Langevin model, however, for same cases the recombination of holes and electrons do not occur instantaneously as they meet and therefore the model fails for describing the charge kinetics. Consequently, $\beta / \beta_{\mathrm{L}} \sim 1$ indicates a Langevin behavior, whereas $\beta / \beta_{\mathrm{L}}<1$ describes devices with non-Langevin recombination. We obtain $\beta / \beta_{\mathrm{L}}$ values of $0.19,0.07$ and 0.44 for PM6:Y6, PM6:O-IDTBR:Y6 1:0.15:0.85 and PM6:O-IDTBR:Y6 1:0.25:0.75, respectively, which clearly reflects the superior transport properties and reduced charge recombination of $15 \%$ ternary blend compared to other devices.

We investigated the morphology of binary and ternary blends via Transmission Electron Microscopy (TEM) and Atomic Force Microscopy (AFM). As previously reported, PM6:Y6 blend exhibits nanofibrous structure (Figure 5a). Upon addition of O-IDTBR $(15 \% \mathrm{w} / \mathrm{w})$ (Figure 5b) the nanofibrous structure are still evident, however, the high miscibility of OIDTBR and Y6 makes it challenging to discern a non-uniform segregation between the components. Whereas, higher loading of O-IDTBR $(25 \% \mathrm{w} / \mathrm{w})$ (Figure 5c) features higher phase segregation due to the higher crystallinity of the third component. Moreover, we calculated similar root mean square (RMS) values for PM6:Y6 1:1, PM6:O-IDTBR:Y6 1:0.15:0.85 and PM6:O-IDTBR:Y6 1:0.25:0.75 film of $2.1 \mathrm{~nm}, 2.3 \mathrm{~nm}$ and $2.7 \mathrm{~nm}$, respectively (Figure S15).

High efficiency organic solar cell devices should also possess photostability and durability under various environmental conditions. However, a device strategy to obtain these requirements is often lacking in the field of OPV. The ternary strategy has been adopted as a powerful method to stabilize binary blend morphologies by, for instance, suppressing the crystallization of NFAs upon the addition of partially miscible third component. ${ }^{29}$ Encouraged by the preferential miscibility of O-IDTBR with Y6, along with improved photovoltaic performances, we studied the operational photostability of binary and ternary devices thoroughly. Figure 5d exhibits the time evolution of PCE values of binary and ternary devices under full solar spectrum irradiation. Notably, the addition of $15 \%$ O-IDTBR leads to 
improved photostability, with a negligible PCE drop in the course of 225 hours (Figure S16), whereas PM6:Y6 binary cells suffer from a severe PCE reduction of over $60 \%$ governed by $V_{\text {oc }}$ losses (Figure S17). The substantial decrease in $V_{\text {oc }}$ can be originated from light-induced trap states/defects. Thus, we measured the $J-V$ characteristics of the degraded devices under different light levels to reveal the presence of traps in the devices (inset Figure 5d). We calculated a slope of $1.31 k \mathrm{~T} / q$ and $1.07 k \mathrm{~T} / q$ for PM6:Y6, PM6:O-IDTBR:Y6 1:0.15:0.85 for degraded solar cells, confirming that the $V_{\text {oc }}$ drop in binary devices are due to light-induced traps, whereas the addition of the crystalline O-IDTBR makes the ternary devices resilient against photodegradation.

In conclusion, we report a design strategy for high efficiency and photostable NFA devices using ternary concept and elaborate on the increased photostability in ternary devices compared to binaries. We selected three NFAs with different EA and structural properties and found that the most crystalline third component, O-IDTBR, is selectively miscible within the acceptor phase resulting in new mixed domains leading to in an increased PCE of $16.63 \%$ and a high FF of 0.76 compared to binary PM6:Y6 devices. We found that the addition of OIDTBR, as a third component, improves charge carrier mobility up to $1.2 \times 10^{-3} \mathrm{~cm}^{2} \mathrm{~V}^{-1} \mathrm{~s}^{-1}$ independent of the applied electrical field, suggesting a more ordered transport in ternary devices in line with the $\beta / \beta_{\mathrm{L}}$ value of 0.07 . We showed that using a ternary concept can be used not only to reduce the trap-assisted recombination in binary devices and enhance the PCE but also to deactivate light-induced traps upon full sun illumination stress. As shown, ternary devices showed an almost unchanged operational stability with a PCE of about $16 \%$ over 225 hours under continuous metal-halide lamp illumination. These results suggest that the ternary strategy can be adopted not only for record efficiency but also to tackle lightinduced degradation in OPV and therefore a beneficial strategy to reduce the efficiencystability gap towards scale-up. 


\section{Acknowledgements}

This publication is based upon work supported by the King Abdullah University of Science and Technology (KAUST) Office of Sponsored Research (OSR) under Award No: OSR2018-CARF/CCF-3079. H.-L. Yip acknowledges the Ministry of Science and Technology (No. 2019YFA0705900) for financial support. N.Gasparini and S.H.K. Paleti contributed equally in this work.

\section{Supporting Information Available.}

Fabrication and Characterization of OSCs, J-Vs characteristics and photovoltaic parameters, optoelectronical measurements conditions and data, DSC, contact angle characterizations details and data, AFM images, photo-CELIV, TPC measurements, GIWAXS measurements. 


\section{References}

(1) Wadsworth, A.; Moser, M.; Marks, A.; Little, M. S.; Gasparini, N.; Brabec, C. J.;

Baran, D.; McCulloch, I. Critical Review of the Molecular Design Progress in Non-

Fullerene Electron Acceptors towards Commercially Viable Organic Solar Cells. Chem.

Soc. Rev. 2019, 48 (6), 1596-1625.

(2) Hou, J.; Inganäs, O.; Friend, R. H.; Gao, F. Organic Solar Cells Based on Non-

Fullerene Acceptors. Nat. Mater. 2018, 17 (2), 119-128.

(3) Zhang, J.; Tan, H. S.; Guo, X.; Facchetti, A.; Yan, H. Material Insights and Challenges for Non-Fullerene Organic Solar Cells Based on Small Molecular Acceptors. Nat.

Energy 2018, 3 (9), 720-731.

(4) Cui, Y.; Yao, H.; Zhang, J.; Zhang, T.; Wang, Y.; Hong, L.; Xian, K.; Xu, B.; Zhang, S.; Peng, J.; Wei, Z.; Gao, F.; Hou, J. Over 16\% Efficiency Organic Photovoltaic Cells Enabled by a Chlorinated Acceptor with Increased Open-Circuit Voltages. Nat.

Commun. 2019, 10 (1), 2515.

(5) Xu, X.; Feng, K.; Bi, Z.; Ma, W.; Zhang, G.; Peng, Q. Single-Junction Polymer Solar Cells with $16.35 \%$ Efficiency Enabled by a Platinum(II) Complexation Strategy. $A d v$. Mater. 2019, 1901872 (Ii), 1901872.

(6) Gao, H.; Sun, Y.; Cai, Y.; Wan, X.; Meng, L.; Ke, X.; Li, S.; Zhang, Y.; Xia, R.; Zheng, N.; Xie, Z.; Li, C.; Zhang, M.; Yip, H.; Cao, Y.; Chen, Y. Achieving Both Enhanced Voltage and Current through Fine-Tuning Molecular Backbone and Morphology Control in Organic Solar Cells. Adv. Energy Mater. 2019, 9 (27), 1901024.

(7) Song, X.; Gasparini, N.; Ye, L.; Yao, H.; Hou, J.; Ade, H.; Baran, D. Controlling Blend Morphology for Ultrahigh Current Density in Nonfullerene Acceptor-Based Organic Solar Cells. ACS Energy Lett. 2018, 3 (3), 669-676.

(8) Kirchartz, T.; Kaienburg, P.; Baran, D. Figures of Merit Guiding Research on Organic 
Solar Cells. J. Phys. Chem. C 2018, 122 (11), 5829-5843.

(9) Gasparini, N.; Salleo, A.; McCulloch, I.; Baran, D. The Role of the Third Component in Ternary Organic Solar Cells. Nat. Rev. Mater. 2019, 4 (4), 229-242.

(10) Qian, D.; Zheng, Z.; Yao, H.; Tress, W.; Hopper, T. R.; Chen, S.; Li, S.; Liu, J.; Chen, S.; Zhang, J.; Liu, X. K.; Gao, B.; Ouyang, L.; Jin, Y.; Pozina, G.; Buyanova, I. A.;

Chen, W. M.; Inganäs, O.; Coropceanu, V.; Bredas, J. L.; Yan, H.; Hou, J.; Zhang, F.;

Bakulin, A. A.; Gao, F. Design Rules for Minimizing Voltage Losses in High-

Efficiency Organic Solar Cells. Nat. Mater. 2018, 17 (8), 703-709.

(11) Zhou, Z.; Xu, S.; Song, J.; Jin, Y.; Yue, Q.; Qian, Y.; Liu, F.; Zhang, F.; Zhu, X. HighEfficiency Small-Molecule Ternary Solar Cells with a Hierarchical Morphology Enabled by Synergizing Fullerene and Non-Fullerene Acceptors. Nat. Energy 2018, 3 (11), 952-959.

(12) Yu, R.; Yao, H.; Cui, Y.; Hong, L.; He, C.; Hou, J. Improved Charge Transport and Reduced Nonradiative Energy Loss Enable Over 16\% Efficiency in Ternary Polymer Solar Cells. Adv. Mater. 2019, 31 (36), 1902302.

(13) Liu, T.; Luo, Z.; Fan, Q.; Zhang, G.; Zhang, L.; Gao, W.; Guo, X.; Ma, W.; Zhang, M.; Yang, C.; Li, Y.; Yan, H. Use of Two Structurally Similar Small Molecular Acceptors Enabling Ternary Organic Solar Cells with High Efficiencies and Fill Factors. Energy Environ. Sci. 2018, 11 (11), 3275-3282.

(14) Song, X.; Gasparini, N.; Nahid, M. M.; Paleti, S. H. K.; Wang, J.; Ade, H.; Baran, D. Dual Sensitizer and Processing-Aid Behavior of Donor Enables Efficient Ternary Organic Solar Cells. Joule 2019, 3 (3), 846-857.

(15) Li, K.; Wu, Y.; Tang, Y.; Pan, M.; Ma, W.; Fu, H.; Zhan, C.; Yao, J. Ternary Blended Fullerene-Free Polymer Solar Cells with 16.5\% Efficiency Enabled with a Higher-LUMO-Level Acceptor to Improve Film Morphology. Adv. Energy Mater. 2019, 9 (33), 1901728. 
(16) Meng, L.; Zhang, Y.; Wan, X.; Li, C.; Zhang, X.; Wang, Y.; Ke, X.; Xiao, Z.; Ding, L.; Xia, R.; Yip, H.-L.; Cao, Y.; Chen, Y. Organic and Solution-Processed Tandem Solar Cells with 17.3\% Efficiency. Science (80-. ). 2018, 361 (6407), 1094-1098.

(17) Li, Y.; Lin, J.-D.; Liu, X.; Qu, Y.; Wu, F.-P.; Liu, F.; Jiang, Z.-Q.; Forrest, S. R. NearInfrared Ternary Tandem Solar Cells. Adv. Mater. 2018, 30 (45), 1804416.

(18) Che, X.; Li, Y.; Qu, Y.; Forrest, S. R. High Fabrication Yield Organic Tandem Photovoltaics Combining Vacuum- and Solution-Processed Subcells with 15\% Efficiency. Nat. Energy 2018, 3 (5), 422-427.

(19) Yuan, J.; Zhang, Y.; Zhou, L.; Zhang, G.; Yip, H.; Lau, T.; Lu, X.; Zhu, C.; Peng, H.; Johnson, P. A.; Leclerc, M.; Cao, Y.; Ulanski, J.; Li, Y.; Zou, Y. Single-Junction Organic Solar Cell with over 15\% Efficiency Using Fused-Ring Acceptor with Electron-Deficient Core. Joule 2019, 3 (4), 1140-1151.

(20) Yao, H.; Cui, Y.; Yu, R.; Gao, B.; Zhang, H.; Hou, J. Design, Synthesis, and Photovoltaic Characterization of a Small Molecular Acceptor with an Ultra-Narrow Band Gap. Angew. Chemie Int. Ed. 2017, 56 (11), 3045-3049.

(21) Zhao, W.; Li, S.; Yao, H.; Zhang, S.; Zhang, Y.; Yang, B.; Hou, J. Molecular Optimization Enables over 13\% Efficiency in Organic Solar Cells. J. Am. Chem. Soc. 2017, 139 (21), 7148-7151.

(22) Baran, D.; Kirchartz, T.; Wheeler, S.; Dimitrov, S.; Abdelsamie, M.; Gorman, J.; Ashraf, R. S.; Holliday, S.; Wadsworth, A.; Gasparini, N.; Kaienburg, P.; Yan, H.; Amassian, A.; Brabec, C. J.; Durrant, J. R.; McCulloch, I. Reduced Voltage Losses Yield 10\% Efficient Fullerene Free Organic Solar Cells with $>1$ V Open Circuit Voltages. Energy Environ. Sci. 2016, 9 (12), 3783-3793.

(23) Yuan, J.; Huang, T.; Cheng, P.; Zou, Y.; Zhang, H.; Yang, J. L.; Chang, S.-Y.; Zhang, Z.; Huang, W.; Wang, R.; Meng, D.; Gao, F.; Yang, Y. Enabling Low Voltage Losses and High Photocurrent in Fullerene-Free Organic Photovoltaics. Nat. Commun. 2019, 
$10(1), 570$.

(24) Du, X.; Heumueller, T.; Gruber, W.; Classen, A.; Unruh, T.; Li, N.; Brabec, C. J.

Efficient Polymer Solar Cells Based on Non-Fullerene Acceptors with Potential Device Lifetime Approaching 10 Years. Joule 2019, 3 (1), 215-226.

(25) Baran, D.; Gasparini, N.; Wadsworth, A.; Tan, C. H.; Wehbe, N.; Song, X.; Hamid, Z.;

Zhang, W.; Neophytou, M.; Kirchartz, T.; Brabec, C. J.; Durrant, J. R.; McCulloch, I.

Robust Nonfullerene Solar Cells Approaching Unity External Quantum Efficiency

Enabled by Suppression of Geminate Recombination. Nat. Commun. 2018, 9 (1), 2059.

(26) Heumueller, T.; Burke, T. M.; Mateker, W. R.; Sachs-Quintana, I. T.; Vandewal, K.;

Brabec, C. J.; McGehee, M. D. Disorder-Induced Open-Circuit Voltage Losses in

Organic Solar Cells During Photoinduced Burn-In. Adv. Energy Mater. 2015, 5 (14), 1500111.

(27) Gasparini, N.; Salvador, M.; Strohm, S.; Heumueller, T.; Levchuk, I.; Wadsworth, A.;

Bannock, J. H.; de Mello, J. C.; Egelhaaf, H.-J.; Baran, D.; McCulloch, I.; Brabec, C. J. Burn-in Free Nonfullerene-Based Organic Solar Cells. Adv. Energy Mater. 2017, 7 (19), 1700770.

(28) Ye, L.; Li, S.; Liu, X.; Zhang, S.; Ghasemi, M.; Xiong, Y.; Hou, J.; Ade, H. Quenching to the Percolation Threshold in Organic Solar Cells. Joule 2019, 3 (2), 443-458.

(29) Zhu, Y.; Gadisa, A.; Peng, Z.; Ghasemi, M.; Ye, L.; Xu, Z.; Zhao, S.; Ade, H. Rational Strategy to Stabilize an Unstable High-Efficiency Binary Nonfullerene Organic Solar Cells with a Third Component. Adv. Energy Mater. 2019, 9 (20), 1900376.

(30) de Zerio, A. D.; Müller, C. Glass Forming Acceptor Alloys for Highly Efficient and Thermally Stable Ternary Organic Solar Cells. Adv. Energy Mater. 2018, 8 (28), 1702741.

(31) Holliday, S.; Ashraf, R. S.; Wadsworth, A.; Baran, D.; Yousaf, S. A.; Nielsen, C. B.; Tan, C.-H.; Dimitrov, S. D.; Shang, Z.; Gasparini, N.; Alamoudi, M.; Laquai, F.; 
Brabec, C. J.; Salleo, A.; Durrant, J. R.; McCulloch, I. High-Efficiency and Air-Stable P3HT-Based Polymer Solar Cells with a New Non-Fullerene Acceptor. Nat. Commun. 2016, 7, 11585.

(32) Baran, D.; Ashraf, R. S.; Hanifi, D. A.; Abdelsamie, M.; Gasparini, N.; Röhr, J. A.; Holliday, S.; Wadsworth, A.; Lockett, S.; Neophytou, M.; Emmott, C. J. M.; Nelson, J.; Brabec, C. J.; Amassian, A.; Salleo, A.; Kirchartz, T.; Durrant, J. R.; McCulloch, I. Reducing the Efficiency-Stability-Cost Gap of Organic Photovoltaics with Highly Efficient and Stable Small Molecule Acceptor Ternary Solar Cells. Nat. Mater. 2017, $16(3), 363-369$.

(33) Zhan, L.; Li, S.; Lau, T.; Cui, Y.; Lu, X.; Shi, M.; Li, C.-Z.; Li, H.; Hou, J.; Chen, H. Over 17\% Efficiency Ternary Organic Solar Cells Enabled by Two Non-Fullerene Acceptors Working in an Alloy-like Model. Energy Environ. Sci. 2020, 13 (2), 635645.

(34) Cheng, P.; Wang, J.; Zhang, Q.; Huang, W.; Zhu, J.; Wang, R.; Chang, S.-Y.; Sun, P.; Meng, L.; Zhao, H.; Cheng, H.-W.; Huang, T.; Liu, Y.; Wang, C.; Zhu, C.; You, W.; Zhan, X.; Yang, Y. Unique Energy Alignments of a Ternary Material System toward High-Performance Organic Photovoltaics. Adv. Mater. 2018, 30 (28), 1801501.

(35) Gasparini, N.; Jiao, X.; Heumueller, T.; Baran, D.; Matt, G. J.; Fladischer, S.; Spiecker, E.; Ade, H.; Brabec, C. J.; Ameri, T. Designing Ternary Blend Bulk Heterojunction Solar Cells with Reduced Carrier Recombination and a Fill Factor of 77\%. Nat. Energy 2016, 1 (9), 16118.

(36) Ye, L.; Collins, B. A.; Jiao, X.; Zhao, J.; Yan, H.; Ade, H. Miscibility-Function Relations in Organic Solar Cells: Significance of Optimal Miscibility in Relation to Percolation. Adv. Energy Mater. 2018, 8 (28), 1703058.

(37) Gasparini, N.; Kahmann, S.; Salvador, M.; Perea, J. D.; Sperlich, A.; Baumann, A.; Li, N.; Rechberger, S.; Spiecker, E.; Dyakonov, V.; Portale, G.; Loi, M. A.; Brabec, C. J.; 
Ameri, T. Favorable Mixing Thermodynamics in Ternary Polymer Blends for Realizing High Efficiency Plastic Solar Cells. Adv. Energy Mater. 2019, 9 (19), 1803394.

(38) Perea, J. D.; Langner, S.; Salvador, M.; Sanchez-Lengeling, B.; Li, N.; Zhang, C.; Jarvas, G.; Kontos, J.; Dallos, A.; Aspuru-Guzik, A.; Brabec, C. J. Introducing a New Potential Figure of Merit for Evaluating Microstructure Stability in Photovoltaic Polymer-Fullerene Blends. J. Phys. Chem. C 2017, 121 (33), 18153-18161.

(39) Cheng, P.; Li, Y.; Zhan, X. Efficient Ternary Blend Polymer Solar Cells with IndeneC60 Bisadduct as an Electron-Cascade Acceptor. Energy Environ. Sci. 2014, 7 (6), 2005.

(40) Gasparini, N.; Lucera, L.; Salvador, M.; Prosa, M.; Spyropoulos, G. D.; Kubis, P.; Egelhaaf, H.-J.; Brabec, C. J.; Ameri, T. High-Performance Ternary Organic Solar Cells with Thick Active Layer Exceeding 11\% Efficiency. Energy Environ. Sci. 2017, $10(4), 885-892$.

(41) Mandoc, M. M.; Kooistra, F. B.; Hummelen, J. C.; De Boer, B.; Blom, P. W. M. Effect of Traps on the Performance of Bulk Heterojunction Organic Solar Cells. Appl. Phys. Lett. 2007, 91 (2007), 2005-2008.

(42) Cowan, S. R.; Roy, A.; Heeger, A. J. Recombination in Polymer-Fullerene Bulk Heterojunction Solar Cells. Phys. Rev. B 2010, 82 (24), 245207.

(43) Song, X.; Gasparini, N.; Nahid, M. M.; Paleti, S. H. K.; Li, C.; Li, W.; Ade, H.; Baran, D. Efficient DPP Donor and Nonfullerene Acceptor Organic Solar Cells with High Photon-to-Current Ratio and Low Energetic Loss. Adv. Funct. Mater. 2019, 29 (34), 1902441.

(44) Street, R. a. Localized State Distribution and Its Effect on Recombination in Organic Solar Cells. Phys. Rev. B 2011, 84 (7), 075208.

(45) Stephen, M.; Genevičius, K.; Juška, G.; Arlauskas, K.; Hiorns, R. C. Charge Transport 
and Its Characterization Using Photo-CELIV in Bulk Heterojunction Solar Cells. Polym. Int. 2017, 66 (1), 13-25.

(46) Gasparini, N.; Salvador, M.; Heumueller, T.; Richter, M.; Classen, A.; Shrestha, S.; Matt, G. J.; Holliday, S.; Strohm, S.; Egelhaaf, H.-J.; Wadsworth, A.; Baran, D.; McCulloch, I.; Brabec, C. J. Polymer:Nonfullerene Bulk Heterojunction Solar Cells with Exceptionally Low Recombination Rates. Adv. Energy Mater. 2017, 7 (22), 1701561.

(47) Song, X.; Gasparini, N.; Nahid, M. M.; Chen, H.; Macphee, S. M.; Zhang, W.; Norman, V.; Zhu, C.; Bryant, D.; Ade, H.; McCulloch, I.; Baran, D. A Highly Crystalline FusedRing n-Type Small Molecule for Non-Fullerene Acceptor Based Organic Solar Cells and Field-Effect Transistors. Adv. Funct. Mater. 2018, 28 (35), 1802895.

(48) Burke, T. M.; Sweetnam, S.; Vandewal, K.; McGehee, M. D. Beyond Langevin Recombination: How Equilibrium Between Free Carriers and Charge Transfer States Determines the Open-Circuit Voltage of Organic Solar Cells. Adv. Energy Mater. 2015, $5(11), 1500123$. 
a

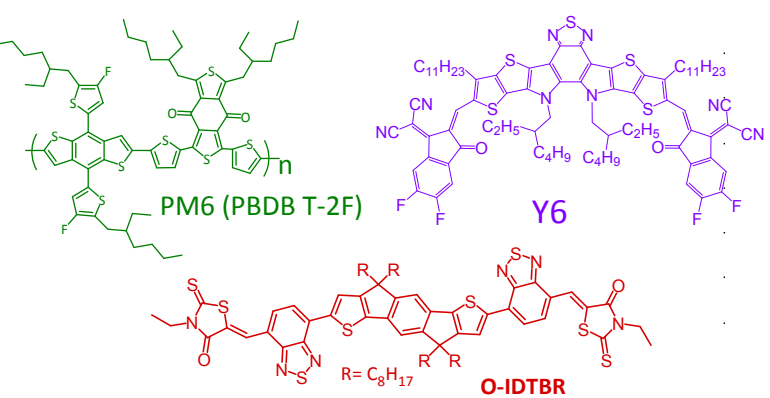

b

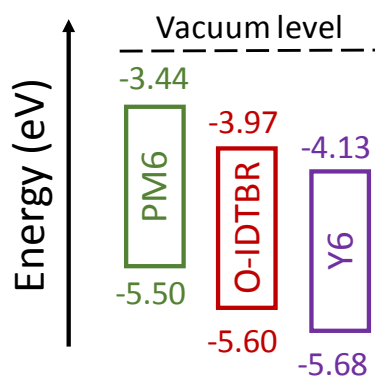

C

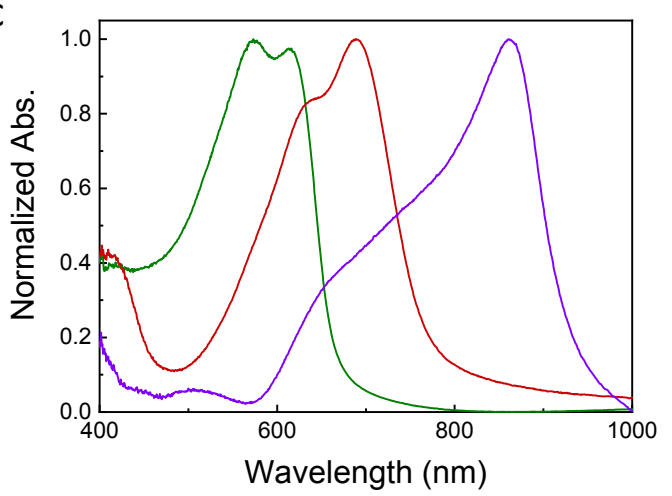

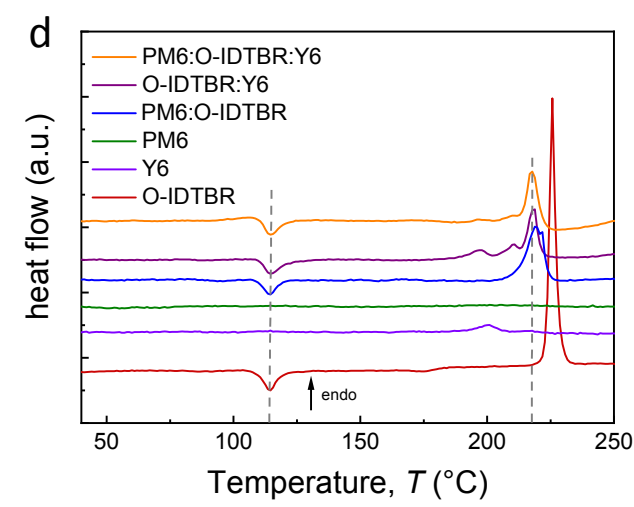

Figure 1. a) Chemical structures, b) energy levels and c) normalized absorbance profiles of the donors and acceptors used in this work. d) Differential scanning calorimetry (DSC) measurements of pristine materials, binary and ternary blends.
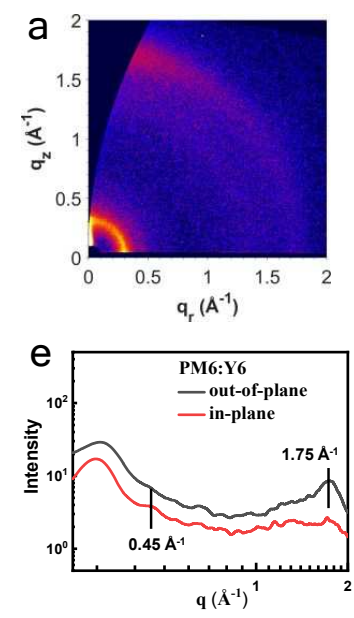
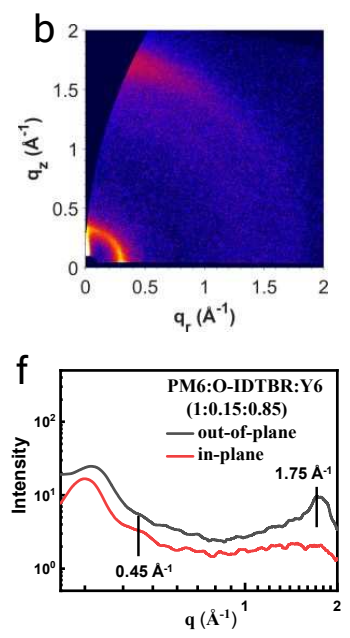
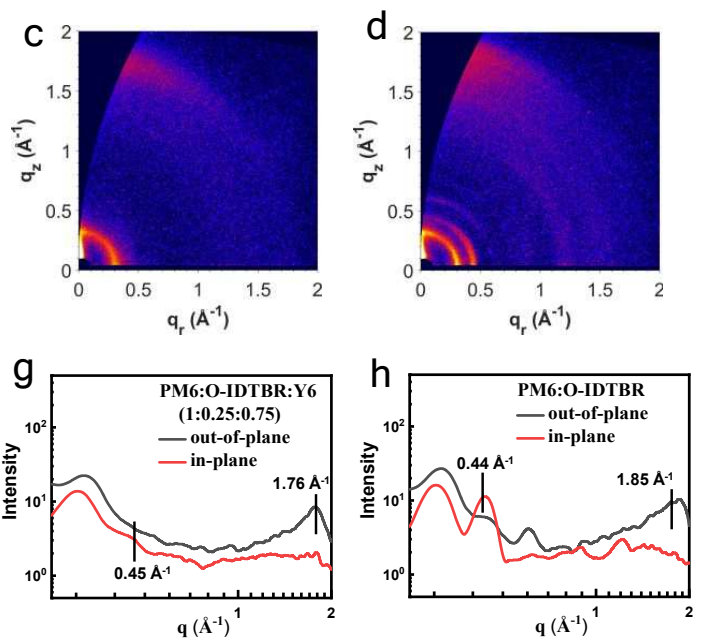

Figure 2. 2D Grazing-incidence wide-angle X-ray scattering, GIWAXS patterns of (a) PM6:Y6 1:1, (b) PM6:O-IDTBR:Y6 1:0.15:0.85, (c) PM6:O-IDTBR:Y6 1:0.25:0.75 and d) 
PM6:O-IDTBR blends. e-h) 1D GIWAXS line cuts of the corresponding blends in the out-ofplane (OOP) direction.
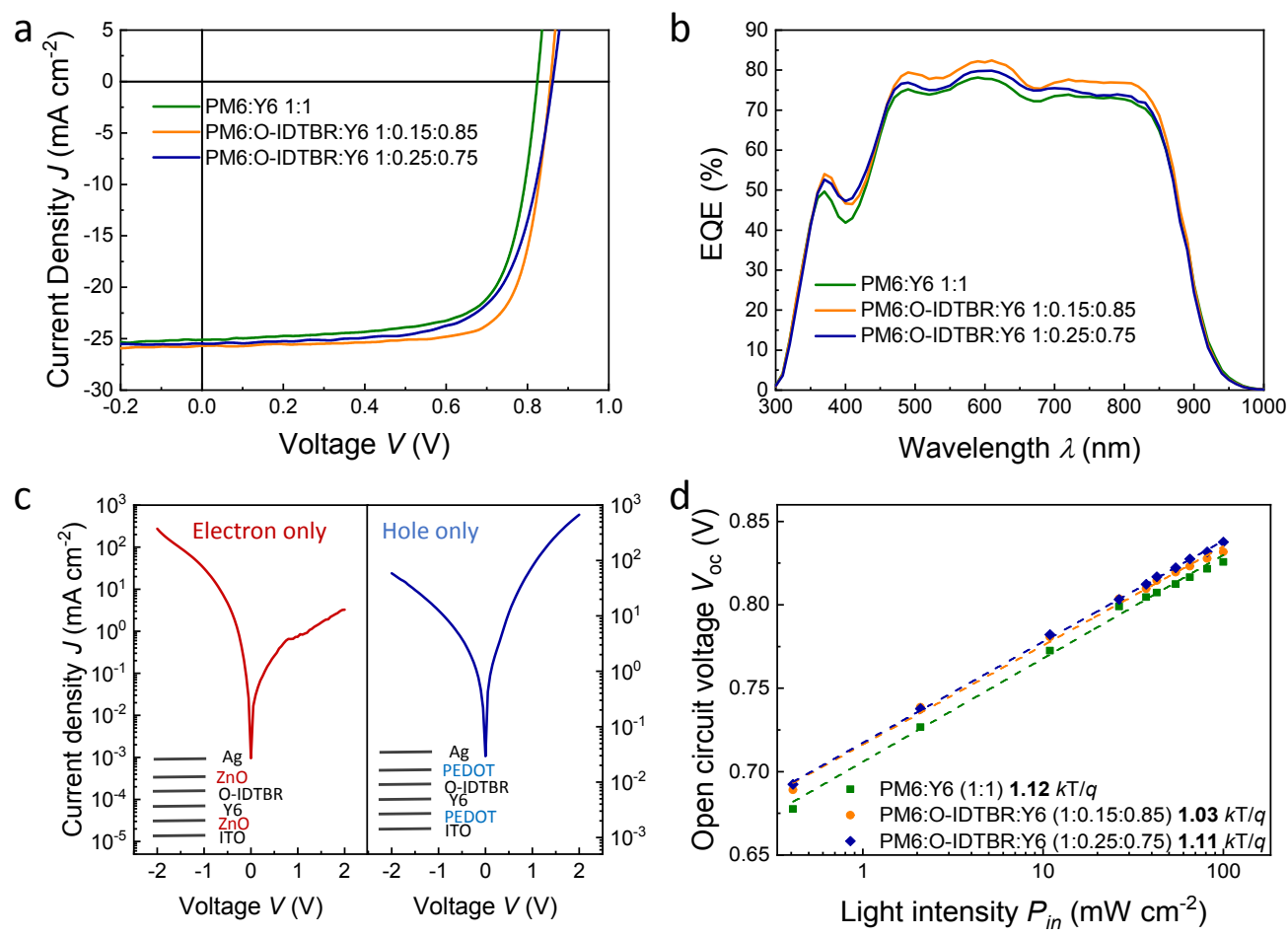

Figure 3. a) Current density-voltage $(J-V)$ characteristics at 1 sun condition for binary and ternary devices and b) their corresponding external quantum efficiency curves. c) $J-V$ curves of electron only and hole only bilayer devices. d) $V_{\text {oc }}$ vs light intensity for binary and ternary devices. 


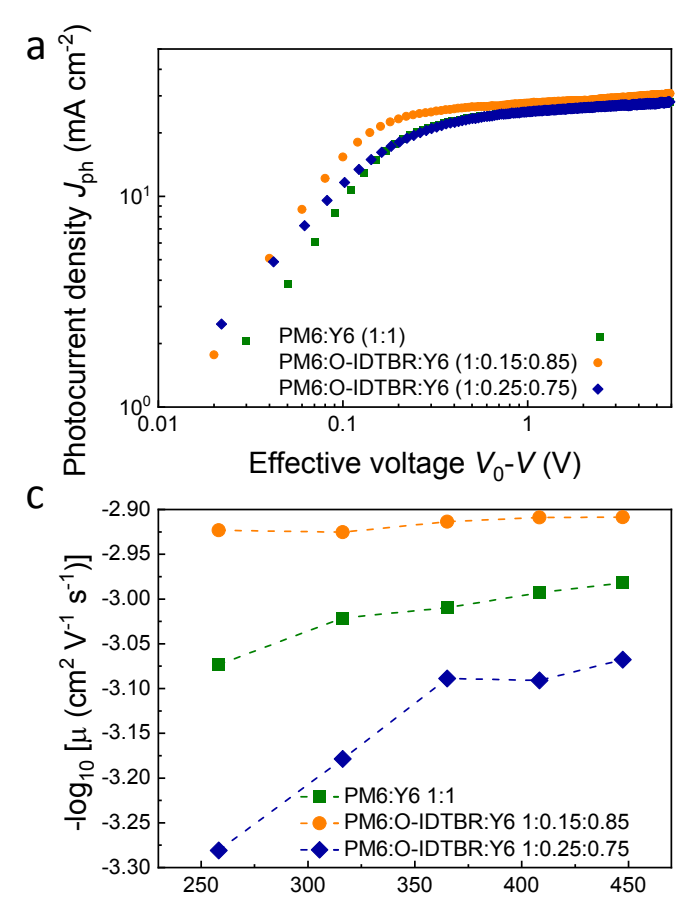

Effective electrical field $E^{1 / 2}\left[\left(\mathrm{~V} \mathrm{~cm}^{-1}\right)^{1 / 2}\right]$

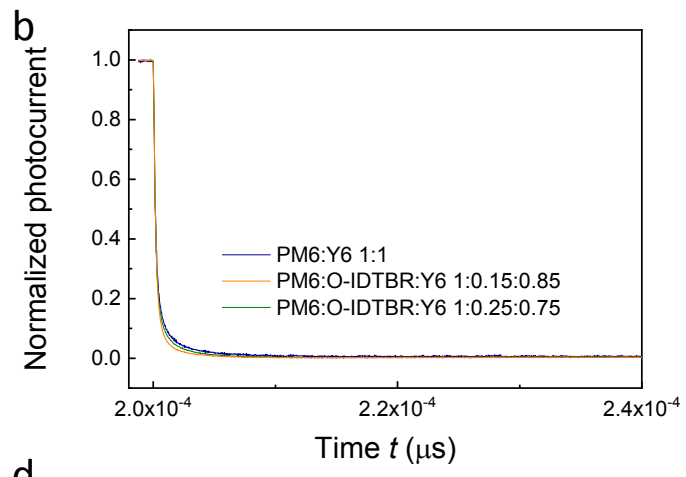

d

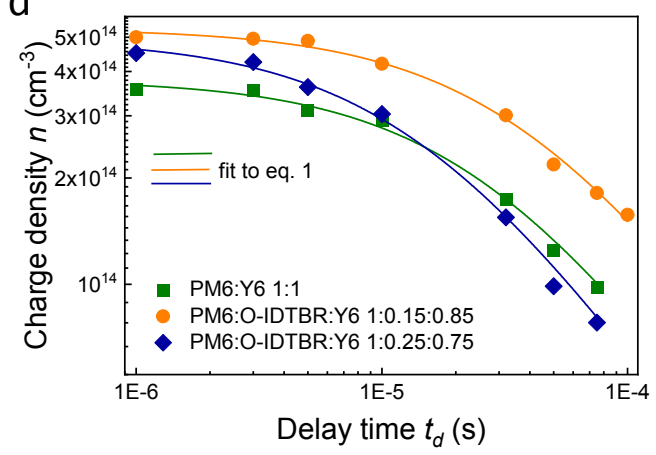

Figure 4. a) Photocurrent density as a function of the effective voltage at 1 sun condition. b) Transient photocurrent traces at 1 sun condition for binary and ternary devices. c) Charge carrier mobility as a function of effective electrical field calculated from photo-CELIV technique. d) Charge density $v s$ delay time calculated from photo-CELIV measurements; the fitted line corresponds to equation 1. 

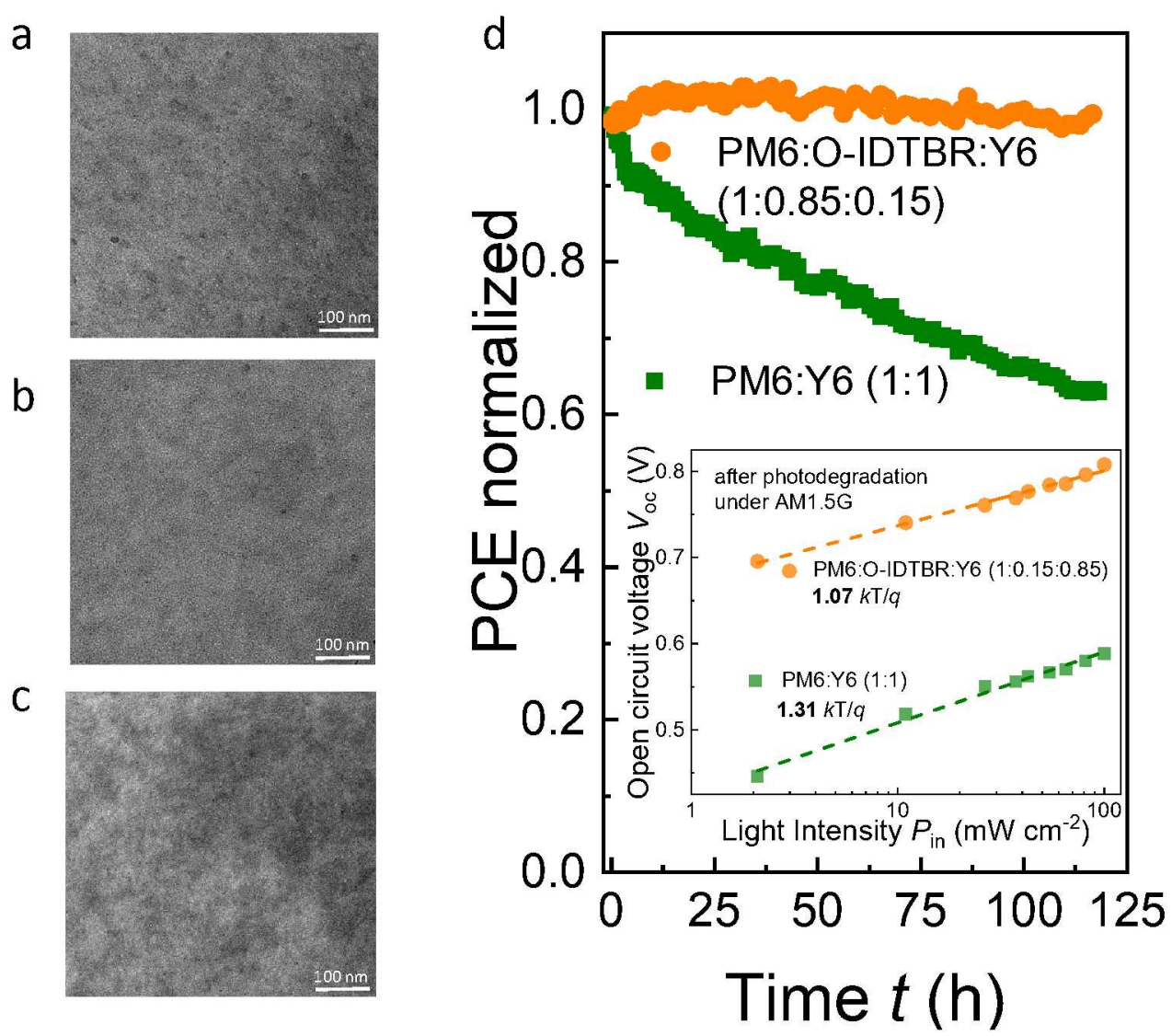

Figure 5. TEM images of PM6:Y6 1:1 (a), PM6:O-IDTBR:Y6 1:0.15:0.85 (b) and PM6:OIDTBR:Y6 1:0.25:0.75 (c) films. d) PCE evolution of binary and ternary devices upon 1 sun illumination stress using a metal halide lamp after $110 \mathrm{~h}$. The inset depicts $V_{\text {oc }}$ vs light intensity for the corresponding degraded devices. 
Table 1 . Key photovoltaics parameters extracted at 1 sun illumination for binary and ternary devices.

\begin{tabular}{|c|c|c|c|c|}
\hline Active layer (ratio) & $\begin{array}{c}V_{o c} \\
(\mathrm{~V})\end{array}$ & $\begin{array}{c}J_{s c} \\
\left(\mathrm{~mA} \mathrm{~cm}^{-2}\right)\end{array}$ & $F F$ & $\begin{array}{c}P C E \\
(\%)\end{array}$ \\
\hline PM6:Y6 1:1 & 0.83 & 25.10 & 0.73 & 15.2 \\
\hline PM6:O-IDTBR:Y6 1:0.15:0.85 & 0.85 & 25.75 & 0.76 & 16.6 \\
\hline PM6:O-IDTBR:Y6 1:0.25:0.75 & 0.86 & 25.46 & 0.69 & 15.1 \\
\hline PM6:EH-IDTBR:Y6 1:0.1:0.9 & 0.84 & 25.9 & 0.71 & 15.5 \\
\hline PM6:O-IDFBR:Y6 1:0.1:0.9 & 0.89 & 25.5 & 0.72 & 16.2 \\
\hline
\end{tabular}

Table 2. Charge transport and recombination parameters for binary and ternary devices.

\begin{tabular}{|c|c|c|c|c|}
\hline PM6:O-IDTBR:Y6 & $\begin{array}{c}\mu \\
\left(\mathrm{cm}^{2} \mathrm{~V}^{-1} \mathrm{~s}^{-1}\right)\end{array}$ & $\begin{array}{c}\beta \\
\left(\mathrm{cm}^{3} \mathrm{~s}^{-1}\right)\end{array}$ & $\begin{array}{c}\beta_{\mathrm{L}} \\
\left(\mathrm{cm}^{3} \mathrm{~s}^{-1}\right)\end{array}$ & $\beta / \beta_{\mathrm{L}}$ \\
\hline $1: 0: 1$ & $8.5 \times 10^{-4}$ & $9.73 \times 10^{-11}$ & $5.13 \times 10^{-10}$ & 0.19 \\
\hline $1: 0.15: 0.85$ & $1.2 \times 10^{-3}$ & $4.75 \times 10^{-11}$ & $7.24 \times 10^{-10}$ & 0.07 \\
\hline $1: 0.25: 0.75$ & $5.2 \times 10^{-4}$ & $1.38 \times 10^{-10}$ & $3.14 \times 10^{-10}$ & 0.44 \\
\hline
\end{tabular}


TOC

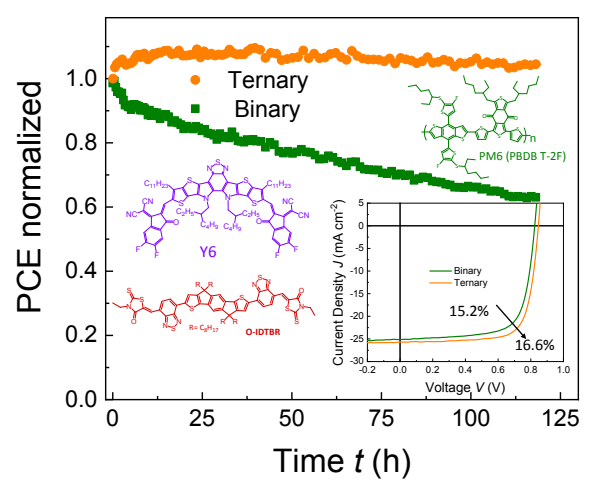

